\title{
Optimized cobalt nanowires for domain wall manipulation imaged by in situ Lorentz microscopy
}

\author{
L. A. Rodríguez, ${ }^{1,2,3,4}$ C. Magén, ${ }^{1,2,3,5, a)}$ E. Snoeck, ${ }^{3,4}$ L. Serrano-Ramón, ${ }^{2,6}$ C. Gatel, ${ }^{3,4}$ \\ R. Córdoba, ${ }^{1,2}$ E. Martínez-Vecino, ${ }^{7}$ L. Torres, ${ }^{7}$ J. M. De Teresa, ${ }^{1,2,6}$ and M. R. Ibarra ${ }^{1,2,3}$ \\ ${ }^{1}$ Laboratorio de Microscopías Avanzadas (LMA), Instituto de Nanociencia de Aragón (INA), \\ Universidad de Zaragoza, 50018 Zaragoza, Spain \\ ${ }^{2}$ Departamento de Física de la Materia Condensada, Universidad de Zaragoza, 50009 Zaragoza, Spain \\ ${ }^{3}$ Transpyrenean Associated Laboratory for Electron Microscopy (TALEM), CEMES-INA, \\ CNRS-Universidad de Zaragoza, Toulouse, France \\ ${ }_{5}^{4}$ CEMES-CNRS 29, rue Jeanne Marvig, B.P. 94347 F-31055, Toulouse Cedex, France \\ ${ }^{5}$ Fundación ARAID, 50004 Zaragoza, Spain \\ ${ }^{6}$ Instituto de Ciencia de Materiales de Aragón (ICMA), Universidad de Zaragoza-CSIC, 50009 Zaragoza, \\ Spain \\ ${ }_{7}^{7}$ Departamento de Física Aplicada, Universidad de Salamanca, 37008 Salamanca, Spain
}

(Received 27 September 2012; accepted 3 January 2013; published online 17 January 2013)

\begin{abstract}
Direct observation of domain wall (DW) nucleation and propagation in focused electron beam induced deposited Co nanowires as a function of their dimensions was carried out by Lorentz microscopy (LTEM) upon in situ application of magnetic field. Optimal dimensions favoring the unambiguous DW nucleation/propagation required for applications were found in 500-nm-wide and 13-nm-thick Co nanowires, with a maximum nucleation field and the largest gap between nucleation and propagation fields. The internal DW structures were resolved using the transport-ofintensity equation formalism in LTEM images and showed that the optimal nanowire dimensions correspond to the crossover between the nucleation of transverse and vortex walls. (C) 2013 American Institute of Physics. [http://dx.doi.org/10.1063/1.4776709]
\end{abstract}

In the last decade, several pioneering works envisaged different strategies to design magnetic information storage, logic devices or sensors based on magnetic domain walls (DW) as functional entities to store, transfer, and process information in ferromagnetic media. ${ }^{1-5}$ These innovative ideas and promising applications have motivated extensive developments of ferromagnetic nanostructures in which DW can be "easily" created and driven either by external magnetic fields and/or spin-polarized currents. ${ }^{6-12} \mathrm{DW}$ in magnetic nanostructures have therefore become a major topic for the research community in the field of Nanomagnetism. The specific DW configuration is the result of the balance between the magnetostatic energy, the magnetocrystalline anisotropy, and the exchange coupling. Therefore, along with the intrinsic properties of the magnetic material, it also depends on the geometry and the dimensions of the nanostructures. ${ }^{13}$ In magnetic nanowires (NWs), the possible DW configurations are either symmetric or asymmetric transverse walls (TWs) or vortex walls (VWs) and they move differently under the application of an external magnetic field or current. ${ }^{14}$ Therefore, a careful analysis of the DW structure of devices as a function of dimensions, geometries, shape of constrictions, etc. is requested to determine the type of magnetic configurations appearing in a given nanostructure and the possibility of tuning and manipulating them upon the application of external magnetic fields or electric currents. ${ }^{6,8,10-12}$

Permalloy (Py) nanostructures have been extensively studied because of their low magnetocrystalline anisotropy, thus allowing the control of its magnetic properties simply

\footnotetext{
${ }^{\text {a) }}$ Author to whom correspondence should be addressed. Electronic mail: cmagend@unizar.es.
}

adjusting its shape anisotropy. Therefore, many Py nanostructures of various shapes and sizes have been synthetized using electron beam or UV lithography processes. ${ }^{1-12,15,16}$ The use of ferromagnetic materials alternative to Py and the development of advanced nanofabrication methods allowing creating magnetic nanostructures of dimensions less than $\sim 100 \mathrm{~nm}$ are however needed to explore their functionalities and possible applications. In the last years, focused electron beam induced deposition (FEBID) technique has demonstrated a capacity to produce high quality nanostructures based on multiple materials. FEBID uses a focused electron beam to locally decompose an organometallic gas injected in the proximity of a substrate and induces the growth of a deposit of the metal on the substrate surface. ${ }^{17,18}$ By tuning multiple growth parameters, high purity deposits can be produced. Particularly cobalt (Co) nanostructures have been obtained with purities up to $95 \%$ so these deposits present optimal properties matching those of the bulk counterpart. ${ }^{19,20}$ Furthermore, as the electron beam producing the deposition can be simply scanned upon the surface, nanostructures of various different shapes and size can be easily produced. ${ }^{20}$ The FEBID spatial resolution is also very high, down to $3 \mathrm{~nm}$ in the case of Pt deposits, ${ }^{21}$ so this technique is capable of producing nanostructures with very fine details such as constrictions, needle shape tips, etc. ${ }^{22}$ In a recent work, high purity Co NWs of lateral size down to $30 \mathrm{~nm}$ were produced by FEBID. ${ }^{20}$ Though the magnetic characterization of this type of systems has been carried out up to now by multiple indirect techniques, such as resistivity, magnetoresistance, Hall effect, magneto-optical Kerr effect, and electron microscopy, ${ }^{19,20,23-25}$ direct observation of the magnetic configurations and magnetization processes with high spatial 
resolution provides additional insights towards an effective DW manipulation. Magnetic force microscopy (MFM) has been used to study the magnetization loops of individual FEBID Co nanostripes and to analyze qualitatively the single- or multidomain nature of the Co nanowire. ${ }^{26}$ MFM can however only give qualitative information on the nature of the DW appearing in these nanostructures. More quantitative characterization of the in-plane magnetization with nanometer-range resolution can be performed using Lorentz transmission electron microscopy (LTEM) in Fresnel mode or Electron Holography. Both have demonstrated the combined possibility of very high spatial resolution with the capability of applying in-situ variation of external constraints such as temperature, ${ }^{27,28}$ magnetic field, ${ }^{6,8,12}$ or electric current. ${ }^{15,16}$ Furthermore, in LTEM phase retrieval by solving the transport-of-intensity equation (TIE) ${ }^{29}$ enables the mapping of the in-plane magnetic induction of the material with the high spatial resolution.

In this letter, we report the complete determination of the nucleation fields in L-shaped FEBID Co NWs as a function of the geometry (thickness and width) and applied magnetic field, analyzed through direct studies of the DW magnetic configurations by means of LTEM in Fresnel mode. We report the most favorable dimensions for the best DW conduit in this magnetic nanostructure. Detailed analysis of the DW configuration and magnetization orientation in these nanostructures were carried out by solving the TIE through focal series of Lorentz images and compared to micromagnetic calculations. We observed that the optimal dimension for DW propagation coincides with the crossover between the nucleation of TW and VW.

L-shaped Co NWs were grown by FEBID in a dual beam system Helios 600 Nanolab from FEI equipped with a gas injector system to inject the organometallic precursor gas $\mathrm{Co}_{2}(\mathrm{CO})_{8}$ inside the deposition chamber. The growth parameters to achieve high purity ( $>90 \%$ ) Co structures have been optimized in previous works and a detailed description can be found elsewhere. ${ }^{19}$ The Co structures have been grown on electron-transparent 50-nm-thick $\mathrm{Si}_{3} \mathrm{~N}_{4}$ membranes, which are suitable for magnetic imaging in transmission experiments using electrons ${ }^{20}$ or $\mathrm{x}$-rays. ${ }^{22}$ The lengths of the two branches of the NWs were fixed for all the objects to $3.5 \mu \mathrm{m}$ and $8.5 \mu \mathrm{m}$ for the short and long branches, respectively. The width $(\mathrm{w})$ and the thickness $(\mathrm{t})$ of the Co nanostructures have been varied through the array, $\mathrm{w}=125,250$, $500,1000 \mathrm{~nm}$ and $\mathrm{t}=5,8,10,13,16,19,22,25,30 \mathrm{~nm}$. Figure 1(b) shows a low magnification TEM image to illustrate the morphology of the L-shaped Co NWs, in this case 500$\mathrm{nm}$-wide and 30-nm-thick. LTEM experiments in Fresnel mode have been performed in a FEI Titan 60-300 Cube equipped with a Lorentz lens for TEM imaging in field-free conditions integrated in an objective lens aberration corrector (CETCOR from CEOS). Magnetic field has been applied to nucleate and manipulate DW by tilting the sample settled in a double tilt TEM specimen holder and slightly exciting the objective lens to produce an in-plane component of magnetic field. ${ }^{30}$ The value and orientation of the in-plane magnetic field have been calibrated with respect to the objective lens excitation and the tilt angles in order to automatically apply the desired value of the in-plane magnetic field compo-
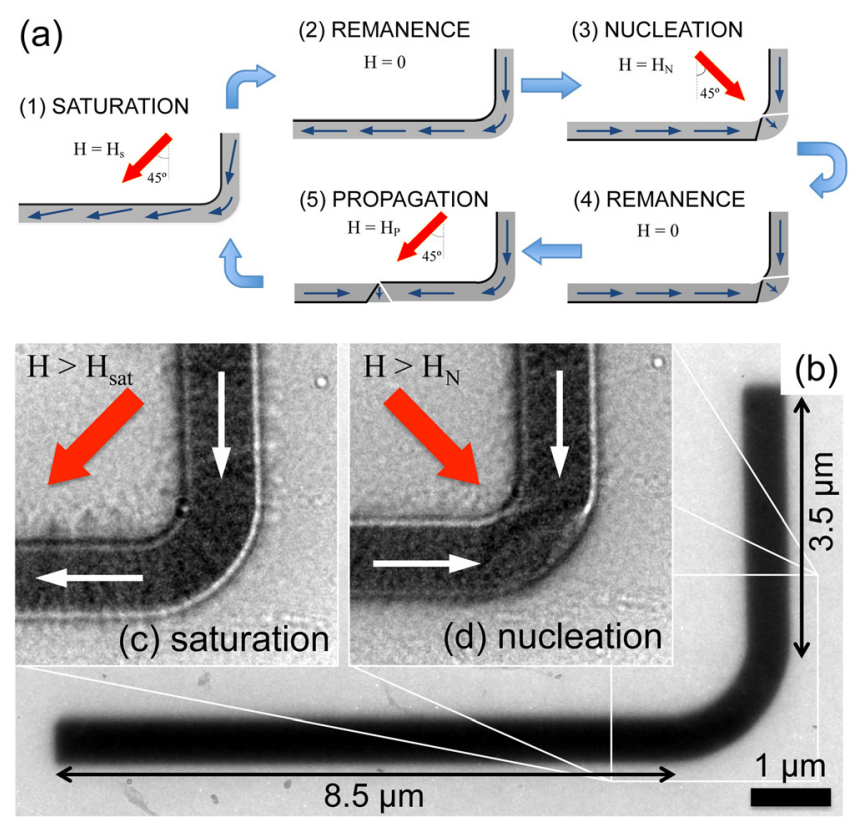

FIG. 1. (a) Schematic representation of the procedure to determine $H_{N}$ and $\mathrm{H}_{\mathrm{P}}$ by magnetic field dependent LTEM (from left to right). (b) Low magnification TEM image of a 500-nm-wide L-shaped FEBID Co nanowire. Insets: Underfocused Lorentz images of the kink when (c) the magnetization is saturated $\left(\mathrm{H}>\mathrm{H}_{\mathrm{sat}}\right)$, and (d) the DW is nucleated $\left(\mathrm{H} \geq \mathrm{H}_{\mathrm{N}}\right)$, where the Fresnel contrast is reversed.

nent in a chosen direction in the plane of the sample. TIE analysis have been performed to retrieve the phase shift of the electron wave at the exit of the sample ${ }^{29}$ and then to reconstruct the in-plane magnetic induction. ${ }^{31}$ Micromagnetic simulations have been performed using the GPMagnet software package ${ }^{32}$ to compare with the experimental data. Real physical dimensions have been used in the models. Inplane magnetic induction maps have been simulated as function of the applied magnetic field in order to determine the remanent magnetic states after DW nucleation.

A complete in-situ analysis of the nucleation field and of the configuration of the DW localized in the curved part of the NWs has been carried out as a function of the magnetic field. Propagation fields have also been measured in selected objects. To nucleate and propagate DW in the round corner of the L-shaped NW, we followed a procedure inspired in Ref. 23. The process is schematized in Figure 1(a). The magnetization is first aligned along the NW by applying a large field of approximately $400 \mathrm{Oe}$ at $45^{\circ}$ with respect to the L-shaped corner, and then the field is decreased down to zero and excited again at $90^{\circ}$ from the previous field direction. The nucleation field $\left(\mathrm{H}_{\mathrm{N}}\right)$ is the field value at which a DW is nucleated in the kink, and it is determined by the direct observation of the contrast changes associated with the presence of a DW in the under-focused LTEM image. The propagation field $\left(\mathrm{H}_{\mathrm{P}}\right)$ can be determined immediately after measuring $\mathrm{H}_{\mathrm{N}}$ by decreasing the magnetic field down to zero and increasing it up at the same initial saturation direction before the nucleation process. $\mathrm{H}_{\mathrm{P}}$ is the magnetic field at which the DW moves out of the kink. In the nanostructures with intermediate dimensions (i.e., $\mathrm{W}=250$ and $500 \mathrm{~nm}$ values), the formation of a DW is deduced from direct observation of lines of bright and dark 
contrast inside the NW kink. This is caused by the deflection of the electron beam by the magnetic structure which depends on the relative orientation of the magnetization as seen in further Figure 4(a). In the case of very narrow objects, the DW structure is difficult to detect as the Fresnel fringes coming from the edges of the nanostructure are overlapping with the DW contrast lines. In such nanowires, $\mathrm{H}_{\mathrm{N}}$ is determined univocally by the observation of the contrast change of the Fresnel fringes along the edges of the NW. When the bright (resp. dark) lines along one side of a branch of the NW changes into dark (resp. bright), a change in the magnetization direction of the long branch has occurred and a DW has appeared. This effect is illustrated in Figures 1(c) and 1(d), in which under-focused LTEM images of a 1000nm-wide Co NW are shown in saturated state [Figure 1(c)] and in remanent state after nucleation [Figure 1(d)]. A continuous bright (dark) contrast in the outer (inner) side of the whole NW is observed in the inset of Figure 1(b) because when the magnetization is saturated the electrons are deflected by the magnetization towards the same side of the NW in both branches. When the magnetization of the long branch is reversed and a head-to-head state is formed in the kink, a contrast reversal of the fringes along the long (horizontal) edges is observed because the magnetization of the two branches deflects the electrons in the opposite direction as observed in Figure 1(d).

Following this strategy, the DW nucleation takes places by a monodomain-type switching of the magnetization in the long branches. In our method, a domain wall is created in the tip of the longer branch and propagates almost instantaneously (after a small increase of the magnetic field) to the curved kink of the NW for $\mathrm{H}_{\text {applied }}=\mathrm{H}_{\mathrm{N}}$. The results of this study are plotted as a function of the width in Figure 2(a). The different experimental points are obtained by averaging the nucleation fields measured in several (up to 5) cycles. The error bars are calculated as the standard deviation of the measured values, and they are due to small hysteretic effects of the objective lens pole pieces and/or small variations in the assessment of the magnetic field value at which the DW is created and propagates. Most NWs present a clear decreasing dependency of $\mathrm{H}_{\mathrm{N}}$ as a function of the width, which qualitatively matches the theoretical dependence with the inverse of the width. However, those with the most reduced dimensions, for instance all those with $\mathrm{w}=125 \mathrm{~nm}$ and for $\mathrm{w}=250 \mathrm{~nm}$ for $\mathrm{t}<13 \mathrm{~nm}$, do not obey the theoretical prediction, presenting significantly diminished $\mathrm{H}_{\mathrm{N}}$ values. This can be explained in terms of a nucleation process dominated by extrinsic factors, such as the dominancy of the surface effects on the DW dynamics of the NW with reduced dimensions. As the surface-to-volume ratio increases, the role of surface roughness in the nucleation/propagation processes becomes more and more important, eventually causing a sudden drop of the $\mathrm{H}_{\mathrm{N}}$ values. These data are plotted differently in Figure 2(b), as a function of the thickness for the different sets of widths. In the NWs of largest and smallest width (1000 nm- and $125 \mathrm{~nm}$-wide), $\mathrm{H}_{\mathrm{N}}$ hardly depends on the thickness $(t)$, being constant around $50 \mathrm{Oe}$ in the case of the $1000 \mathrm{~nm}$-wide NWs and approximately $100 \mathrm{Oe}$ for $\mathrm{w}=125 \mathrm{~nm}$. A progressive increase of $\mathrm{H}_{\mathrm{N}}$ with thickness is observed in the $250 \mathrm{~nm}$-wide NWs from 115 Oe for $\mathrm{t}=5 \mathrm{~nm}$
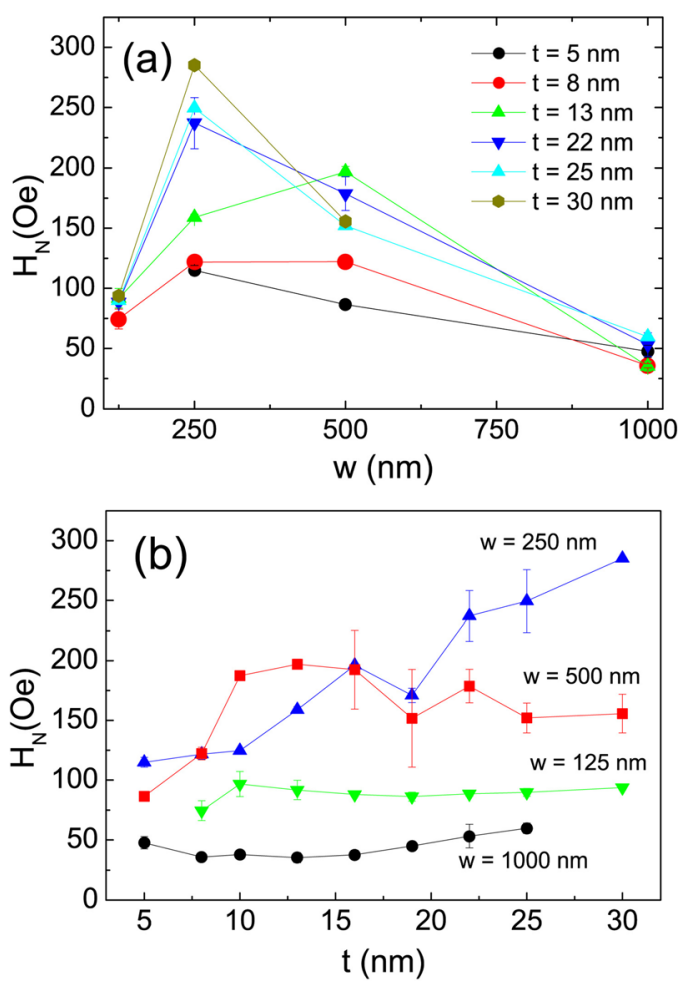

FIG. 2. Dependence of the nucleation fields as a function of (a) the width and (b) the thickness of the nanowires. In (a), some thicknesses are not plotted for the sake of clarity.

up to close to 285 Oe for $\mathrm{t}=30 \mathrm{~nm}$. More interestingly, the $500 \mathrm{~nm}$-wide NWs present a maximum of $\mathrm{H}_{\mathrm{N}}=196$ Oe for $\mathrm{t}=13 \mathrm{~nm}$, whereas in the thinnest NWs $(\mathrm{t}=5 \mathrm{~nm})$ DW nucleates at $86 \mathrm{Oe}$, and at $155 \mathrm{Oe}$ in the thickest ones $(\mathrm{t}=30 \mathrm{~nm})$.

Due to the anomalous $\mathrm{H}_{\mathrm{N}}$ dependence observed, $\mathrm{H}_{\mathrm{P}}$ have been determined for the $500 \mathrm{~nm}$-wide NWs, the result is plotted together with the $\mathrm{H}_{\mathrm{N}}$ values in Fig. 3. In sharp contrast with the $\mathrm{H}_{\mathrm{N}}$ thickness dependence, the propagation field presents a nearly constant value varying from 55 to $65 \mathrm{Oe}$ in the thickness range from 8 to $22 \mathrm{~nm}$. As a result, the optimal thickness $(\mathrm{t}=13 \mathrm{~nm})$ that leads to a maximum nucleation field $\left(\mathrm{H}_{\mathrm{N}}=196 \mathrm{Oe}\right)$ gives rise to the largest difference between the nucleation $\mathrm{H}_{\mathrm{N}}$ and the propagation $\mathrm{H}_{\mathrm{P}}$ fields $(\Delta \mathrm{H})$ of $142 \mathrm{Oe}$. Such a large $\Delta \mathrm{H}$ is important to optimally manipulate the DW for information storage or processing, as

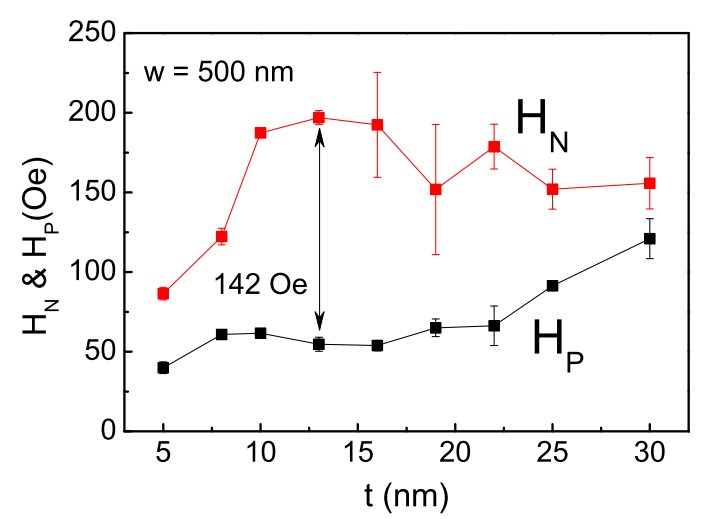

FIG. 3. Comparison between the nucleation fields and the propagation fields for the 500-nm-wide Co nanowires as a function of the thickness. 

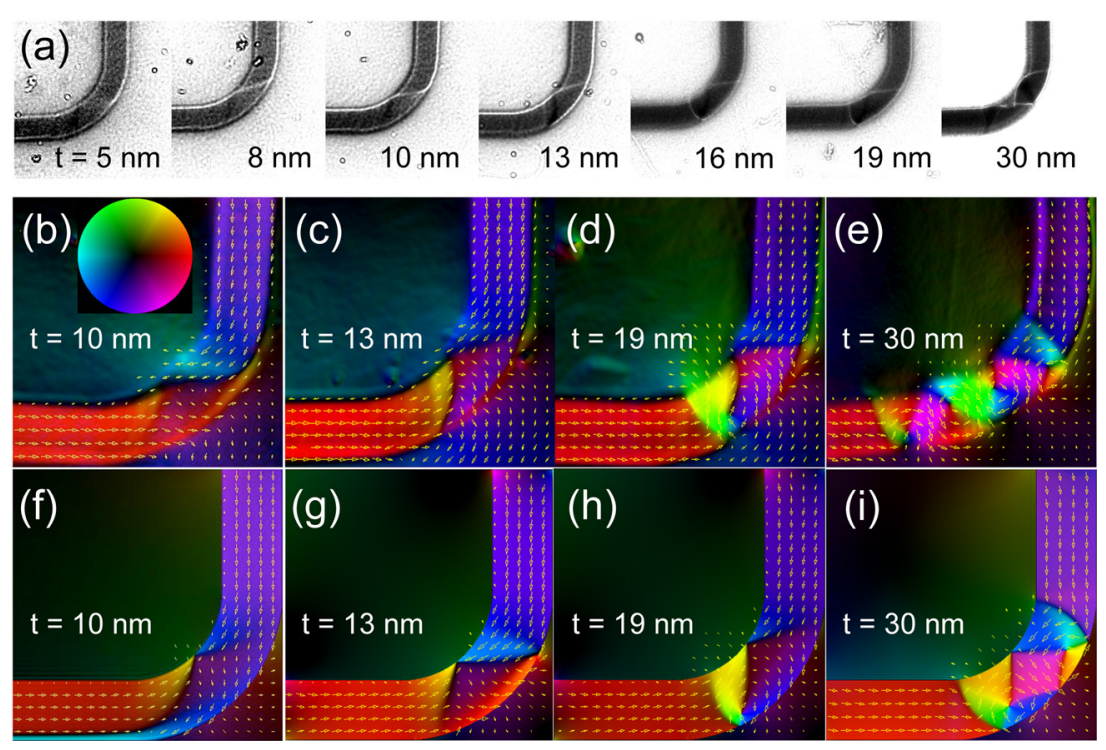

FIG. 4. (a) Sequence of out-of-focus Lorentz images of L-shaped nanowires as a function of the thickness in $500 \mathrm{~nm}$-wide Co NWs. (b)-(e) TIE-reconstructed inplane magnetic induction of a Co NW in the TW regime $(\mathrm{t}=10$ and $13 \mathrm{~nm})$, in the VW regime $(\mathrm{t}=30 \mathrm{~nm})$, with an intermediate state between TW and VW $(\mathrm{w}=19 \mathrm{~nm})$. (f)-(i) Micromagnetic simulations of the NWs reconstructed by TIE. The inset in (b) shows the color code used for the different orientations in all the figures.

it facilitates their independent nucleation and propagation at largely different applied fields. This is a crucial point to minimize the writing/reading errors due to random thermal DW depinning. Furthermore, the set of 500-nm-wide NWs lies on the range of dimensions that still obeys the theoretical prediction for $\mathrm{H}_{\mathrm{N}}$ for intrinsic nucleation processes determined by the exchange and magnetostatic energy. It also increases the tolerance of the magnetic device to small local variations of $\mathrm{H}_{\mathrm{N}}$ and $\mathrm{H}_{\mathrm{P}}$ that may occur during fabrication such as roughness, composition, inhomogeneities, etc. As $500 \mathrm{~nm}$ wide NWs exhibit this anomalous $\mathrm{H}_{\mathrm{N}}$ evolution as function of thickness with the appearance of an optimal $\Delta \mathrm{H}$ value, we focus our studies on the analysis of the DW configurations in these particular NWs. DW have then been analyzed using the TIE analysis of focal series of LTEM images. Figure 4(a) displays a series of defocused LTEM images obtained in the $500 \mathrm{~nm}$-wide NWs for different thickness, which already indicates the formation of two types of domains as a function of thickness, a TW for $\mathrm{t}<\mathrm{t}_{\max }$ and a VW (or multi- $\mathrm{VW}$ ) for $t>t_{\text {max }}$. TIE reconstructions have been carried out to corroborate this fact and provide more detailed information on the magnetic structure nucleated at the kink. This is displayed in Figures 4(b)-4(e), where the color- and arrow-coded TIE reconstruction of the in-plane magnetic induction obtained from LTEM images is reported for "thin" and "thick" NWs, i.e., Fig. 4(b): $t=10 \mathrm{~nm}$, Fig. 4(c): $t=13 \mathrm{~nm}$; Fig. 4(d): $\mathrm{t}=19 \mathrm{~nm}$; Fig. $4(\mathrm{e}): \mathrm{t}=30 \mathrm{~nm}$. It is clearly seen that a TW is formed at $\mathrm{t}=10$ and $13 \mathrm{~nm}$, a VW structure is observed for $\mathrm{t}=19 \mathrm{~nm}$, and a multi-VW configuration is determined at $\mathrm{t}=30 \mathrm{~nm}$. Micromagnetic simulations of the Co nanowires with the same width and thickness have been performed to support our experimental observations. A defect-free model of the nanowires with squared profiles and bulk-like magnetic parameters was used. Simulations were carried out following the same experimental procedure and are depicted in Figs. 4(f)-4(i), the magnetic configuration shown in the images being the remanent state after nucleating the DW. Despite the small differences in the positions of the DW, most likely due to morphological differences between the real and the simulated defect-free nanostructures (roughness, irregularities, small thickness variations), the micromagnetic simulations are in very good agreement with the TIE reconstructions. They demonstrate that the DW nucleated in the curved corner is a TW in the case of thin nanowires (for $\mathrm{t}$ $\leq \mathrm{t}_{\max }$ ), whereas in thick NWs VW or multi-VW structures are pinned close to the corner. These results indicate that the width and thickness of the NW which present the most valuable $\mathrm{H}_{\mathrm{N}}, \mathrm{H}_{\mathrm{P}}$, and $\Delta \mathrm{H}$ fields for application $(\mathrm{w}=500 \mathrm{~nm}$ and $\mathrm{t} \approx 13 \mathrm{~nm}$ ) are also the dimension where a crossover between TW and VW states occurs. ${ }^{14}$

To summarize, nucleation fields $\left(\mathrm{H}_{\mathrm{N}}\right)$ and propagation fields $\left(\mathrm{H}_{\mathrm{P}}\right)$ of domain wall in L-shaped FEBID Co nanostructures have been studied by in situ Lorentz TEM. We evidenced an optimal Co NW dimension of $\mathrm{w}_{\max }=500 \mathrm{~nm}$ width and $\mathrm{t}_{\max }=13 \mathrm{~nm}$ for which the nucleation field is maximal $(197 \pm 4 \mathrm{Oe})$ and the difference between $H_{N}$ and $H_{P}$ is also maximized, $\Delta \mathrm{H}=146 \mathrm{Oe}$. Such large difference between $\mathrm{H}_{\mathrm{N}}$ and $\mathrm{H}_{\mathrm{P}}$ is required for technological applications to guarantee safe and independent nucleation and propagation of DW. This optimal physical dimension coincides to a thickness where the DW switches from a transverse wall configuration (for $\mathrm{t}<\mathrm{t}_{\max }$ ) to a vortex wall type for thicker NW.

This work was supported by Spanish Ministry of Economy and Competitivity through project Nos. MAT2011-28532-C03-01, MAT2011-28532-C03-02, and MAT2011-27553-C02, including FEDER funds, by Junta de Castilla y Leon through the Project SA163A12, and by the European Union Seventh Framework Programme under Grant Agreement 312483-ESTEEM2 (Integrated Infrastructure Initiative-I3) and TRAIN2 project.

${ }^{1}$ T. Ono, H. Miyajima, K. Shigeto, K. Mibu, N. Hosoito, and T. Shinjo, Science 284, 468-470 (1999).

${ }^{2}$ D. Atkinson, D. A. Allwood, G. Xiong, M. D. Cooke, C. C. Faulkner, and R. P. Cowburn, Nature Mater. 2, 85-87 (2003).

${ }^{3}$ D. A. Allwood, G. Xiong, C. C. Faulkner, D. Atkinson, D. Petit, and R. P. Cowburn, Science 309, 1688-1692 (2005).

${ }^{4}$ M. Hayashi, L. Thomas, R. Moriya, C. Rettner, and S. S. P. Parkin, Science 320, 209-211 (2008).

${ }^{5}$ S. S. P. Parkin, M. Hayashi, and L. Thomas, Science (New York, N.Y.) 320, 190-194 (2008). 
${ }^{6}$ D. McGrouther, S. McVitie, J. N. Chapman, and A. Gentils, Appl. Phys. Lett. 91, 022506 (2007).

${ }^{7}$ D. Backes, C. Schieback, M. Kläui, F. Junginger, H. Ehrke, P. Nielaba, U. Rüdiger, L. J. Heyderman, C. S. Chen, T. Kasama, R. E. DuninBorkowski, C. A. F. Vaz, and J. A. C. Bland, Appl. Phys. Lett. 91, 112502 (2007).

${ }^{8}$ C. W. Sandweg, N. Wiese, D. McGrouther, S. J. Hermsdoerfer, H. Schultheiss, B. Leven, S. McVitie, B. Hillebrands, and J. N. Chapman, J. Appl. Phys. 103, 093906 (2008).

${ }^{9}$ M.-Y. Im, L. Bocklage, P. Fischer, and G. Meier, Phys. Rev. Lett. 102 , 147204 (2009).

${ }^{10}$ S. Lepadatu, A. Vanhaverbeke, D. Atkinson, R. Allenspach, and C. H. Marrows, Phys. Rev. Lett. 102, 127203 (2009).

${ }^{11}$ Y. Nakatani, A. Thiaville, and J. Miltat, J. Magn. Magn. Mater. 290-291, 750-753 (2005).

${ }^{12}$ K. J. O'Shea, S. McVitie, J. N. Chapman, and J. M. R. Weaver, Appl. Phys. Lett. 93, 202505 (2008).

${ }^{13}$ R. P. Cowburn, J. Phys. D: Appl. Phys. 33, R1-R16 (2000).

${ }^{14}$ M. Kläui, J. Phys.: Condens. Matter 20, 313001 (2008).

${ }^{15}$ F. Junginger, M. Kläui, D. Backes, U. Rüdiger, T. Kasama, R. E. DuninBorkowski, L. J. Heyderman, C. A. F. Vaz, and J. A. C. Bland, Appl. Phys. Lett. 90, 132506 (2007).

${ }^{16}$ E.-M. Hempe, M. Kläui, T. Kasama, D. Backes, F. Junginger, S. Krzyk, L. J. Heyderman, R. E. Dunin-Borkowski, and U. Rüdiger, Phys. Status Solidi A 204, 3922-3928 (2007).

${ }^{17}$ I. Utke, P. Hoffmann, and J. Melngailis, J. Vac. Sci. Technol. B 26, 1197 (2008).

${ }^{18}$ W. F. van Dorp and C. W. Hagen, J. Appl. Phys. 104, 081301 (2008).

${ }^{19}$ A. Fernández-Pacheco, J. M. De Teresa, R. Córdoba, and M. R. Ibarra, J. Phys. D: Appl. Phys. 42, 055005 (2009).
${ }^{20}$ L. Serrano-Ramón, R. Córdoba, L. A. Rodríguez, C. Magén, E. Snoeck, C. Gatel, I. Serrano, M. R. Ibarra, and J. M. De Teresa, ACS Nano 5, 77817787 (2011).

${ }^{21}$ L. van Kouwen, A. Botman, and C. W. Hagen, Nano Lett. 9, 2149-2152 (2009).

${ }^{22}$ A. Fernández-Pacheco, L. E. Serrano-Ramón, T. Tyliszczak, K. W. Chou, R. Córdoba, A. Szkudlarek, L. O. Brien, C. Kapusta, M. R. Ibarra, and J. M. De Teresa, Nanotechnology 23, 105703 (2012).

${ }^{23}$ A. Fernández-Pacheco, J. M. De Teresa, R. Córdoba, M. R. Ibarra, D. Petit, D. E. Read, L. O'Brien, E. R. Lewis, H. T. Zeng, and R. P. Cowburn, Appl. Phys. Lett. 94, 192509 (2009).

${ }^{24}$ A. Fernández-Pacheco, J. M. De Teresa, A. Szkudlarek, R. Córdoba, M. R. Ibarra, D. Petit, L. O'Brien, H. T. Zeng, E. R. Lewis, D. E. Read, and R. P. Cowburn, Nanotechnology 20, 475704 (2009).

${ }^{25}$ R. Córdoba, R. Fernández-Pacheco, A. Fernández-Pacheco, A. Gloter, C. Magén, O. Stéphan, M. R. Ibarra, and J. M. De Teresa, Nanoscale Res. Lett. 6, 592 (2011).

${ }^{26}$ M. Jaafar, L. Serrano-Ramón, O. Iglesias-Freire, A. Fernández-Pacheco, M. R. Ibarra, J. M. De Teresa, and A. Asenjo, Nanoscale Res. Lett. 6, 407 (2011).

${ }^{27}$ X. Z. Yu, Y. Onose, N. Kanazawa, J. H. Park, J. H. Han, Y. Matsui, N. Nagaosa, and Y. Tokura, Nature 465, 901-904 (2010).

${ }^{28}$ Y. Murakami, H. Kasai, J. J. Kim, S. Mamishin, D. Shindo, S. Mori, and A. Tonomura, Nat. Nanotechnol. 5, 37-41 (2010).

${ }^{29}$ D. Paganin and K. A. Nugent, Phys. Rev. Lett. 80, 2586 (1998).

${ }^{30}$ E. Javon, C. Gatel, A. Masseboeuf, and E. Snoeck, J. Appl. Phys. 107, 09D310 (2010).

${ }^{31}$ V. V. Volkov and Y. Zhu, Ultramicroscopy 98, 271-281 (2004).

${ }^{32}$ L. Lopez-Diaz, D. Aurelio, L. Torres, E. Martinez, M. A. Hernandez-Lopez, J. Gomez, O. Alejos, M. Carpentieri, G. Finocchio, and G. Consolo, J. Phys. D: Appl. Phys. 45, 323001 (2012). 